Senra, K. B., \& Vieira, F. G. D. (2020). Pierre Bourdieu em Marketing e Estudos de Consumo: Estado da arte e agenda de pesquisa. Consumer Behavior Review, 4(3), 199-216.

ISSN: 2526-7884

Editor: Prof. Dr. Marconi Freitas da Costa Journal's e-mail: cbr@ufpe.br
Evaluation: Double blind review

Received: June 08, 2020

Accepted: August 22, 2020

\title{
PIERRE BOURDIEU EM MARKETING E ESTUDOS DE CONSUMO: ESTADO DA ARTE E AGENDA DE PESQUISA
}

Pierre Bourdieu in Marketing and Consumption Studies: State of the art and research agenda

\author{
Karin Borges Senra ${ }^{1}$ \\ ORCID: http://orcid.org/0000-0002-0118-4353 \\ E-mail: karinbsenra@gmail.com \\ Francisco Giovanni David Vieira ${ }^{2}$ \\ ORCID: http://orcid.org/0000-0002-6204-0855 \\ E-mail: fgdvieira@uem.br
}

${ }^{1}$ Pontifícia Universidade Católica do Paraná, Maringá, Brasil

${ }^{2}$ Programa de Pós-Graduação em Administração, Universidade Estadual de Maringá, Maringá, Brasil

\begin{abstract}
Resumo
Diante da aplicabilidade dos conceitos de Pierre Bourdieu para a produção em Marketing e em estudos de consumo, bem como da escassez de estudos dessa natureza vistos ao longo dos últimos anos, este artigo apresenta um panorama dos estudos em Marketing e consumo que utilizam Bourdieu no período de 2008 a 2018, e com base nesses resultados desenvolve uma agenda de pesquisa na área. No levantamento realizado foram encontrados 46 artigos. Como principais resultados verifica-se que os estudos realizados se situam amplamente na área de consumo, possuem predominância de
\end{abstract}

\begin{abstract}
In view of the applicability of Pierre Bourdieu's concepts to research in Marketing and consumption studies, as well as the scarcity of studies of this nature seen over the last few years, this paper presents an overview of the studies in Marketing and consumption studies that has used Bourdieu from 2008 to 2018, and based on these results develops a research agenda in the area. In the survey were found 46 articles. The main results show that the studies carried out are largely in the area of consumption, have a predominance of qualitative methodologies, with interviews
\end{abstract}


metodologias qualitativas, com entrevistas e observação como principais formas de coleta de dados, e análise interpretativa, hermenêutica e de discurso, como principais procedimentos de análise de dados, bem como destacam temas sociais e políticos nos contextos de Marketing e estudos de consumo. Diante dos resultados são feitas proposições de pesquisa, tanto aproveitando a consolidação dessas formas já realizadas, quanto apresentando o potencial que os conceitos de Bourdieu possuem para a pesquisa em Marketing e estudos de consumo. Palavras-chave: Bourdieu. Marketing. Consumo. Agenda de Pesquisa. and observation as main forms of data collection, and interpretative, hermeneutic and discourse analysis, as the main analysis procedures as well as highlight social and political issues in Marketing and consumption studies contexts. Then research proposals are made, both considering the consolidation of these already realized forms, and presenting the potential that the concepts of Bourdieu have for research in Marketing and consumption studies.

Keywords: Bourdieu. Marketing. Consumption. Research Agenda.

This work is licensed under a Creative Commons Attribution 4.0 International License.

\section{INTRODUÇÃO}

Os conceitos desenvolvidos por Bourdieu são utilizados em diversas áreas de pesquisa, com diferentes aplicações. Nos últimos 10 anos destacamos pesquisas em administração (Natt \& Ychikawa, 2012), contabilidade (Farjaudon \& Morales, 2013), educação (English, 2009; McCandless, 2015; Storey, Killian, \& O’Regan, 2017), esportes (Ferry, 2014; Kitchin \& Howe, 2013), estética (Holliday \& Cairnie, 2007), moda (Rafferty, 2011), saúde (Adkins \& Corus, 2009) e entretenimento (Lavie \& Dhoest, 2015), entre outras. Embora muitas vezes caracterizem trabalhos em outras áreas, encontramos diálogos com Marketing, estudos de consumo e seus interesses.

Dessa maneira, partimos do pressuposto de que as teorias de Bourdieu constituem rica fonte teórica para análise de contextos sociais no âmbito de Marketing. Isso pode ser justificado pelo fato de que Bourdieu considera que todo espaço social (campo) analisado é formado por agentes (indivíduos e instituições), dentre os quais, produtores, consumidores e intermediários culturais são parte fundamental na constituição (Bourdieu, 1989; 2007). Em outras palavras, a compreensão de Bourdieu dos espaços sociais é formada pela produção e pelo consumo de bens e serviços, bem como pelas diversas práticas dos agentes envolvidos para se posicionar nesse contexto social. Assim, a atuação de agentes de Marketing pode ser observada e analisada por essa lente teórica.

De modo complementar, a teoria da prática de Bourdieu, incorpora conceitos tratados no seu amplo referencial teórico e pode ser usada como método em conjunto com tais conceitos (Bourdieu, Chamboredon, \& Passeron, 1999). A teoria de Bourdieu sobre espaços sociais foi construída de forma que pudesse ser utilizada não somente para teorizar sobre o funcionamento desses espaços, mas para servir como ferramenta metodológica para compreender os diferentes espaços sociais, o que amplia as possibilidades de utilização no contexto de Marketing e estudos de consumo.

Marketing é uma disciplina que tem início no século XX e se consolida no início do século XXI, sendo composta por diversas escolas de pensamento que envolvem estudos de consumo. Durante o seu desenvolvimento a escola de comportamento do consumidor foi pioneira na utilização de abordagens sociológicas para a construção do conhecimento (Shaw \& Jones, 2005). Nessa escola, uma corrente teórica denominada Consumer Culture Theory (CCT) tem grande representatividade nesse uso, adotando abordagens mais interpretativas, com teorias da sociologia e antropologia, dentre as quais o referencial de Bourdieu faz parte (Askegaard \& Linnet, 2011).

No entanto, embora muito útil para a compreensão de contextos sociais, a literatura de Bourdieu ainda é pouco usada para a compreensão de fenômenos de Marketing e estudos de consumo (Strehlau, 2007). Nesse sentido, concordamos com Anderson (1983) que Marketing e estudos de consumo precisam ser guiados por outras ciências, dentre elas as ciências sociais, da qual a Sociologia e a Antropologia fazem parte. 
Com base nisso, buscamos apresentar o uso feito dos conceitos de Bourdieu nos últimos anos em pesquisas em Marketing e estudos de consumo, e desenvolver uma agenda de pesquisa para que novos pesquisadores possam olhar os espaços sociais sob essa lente teórica, que já se mostrou capaz de trazer novas explicações para os fenômenos de Marketing e estudos de consumo. Dessa forma, podemos contribuir com o campo de pesquisa de Marketing e estudos de consumo a fim de mostrar oportunidades de pesquisa. Após esta introdução, apresentamos os principais conceitos utilizados por Bourdieu, seguidos da metodologia, resultados e análises dos dados. Por fim, desenvolvemos uma agenda de pesquisa em Marketing e estudos de consumo e delineamos as considerações finais do artigo.

\section{PIERRE BOURDIEU}

Ao longo da sua trajetória, Bourdieu elaborou uma série de conceitos que se relacionam e auxiliam nas explicações de fenômenos sociais. Podemos dizer que todos os conceitos que Bourdieu desenvolve se concentram na ideia de campos, sendo essa a base para a compreensão dos espaços sociais.

A teoria dos campos de Bourdieu compreende uma visualização dos espaços sociais como estruturas de relações que formam as práticas dos indivíduos pertencentes a eles. Os espaços sociais considerados campos não são espaços geográficos, mas espaços simbólicos que envolvem os agentes do campo. Os campos possuem uma hierarquia, de forma que cada agente, indivíduo ou instituição, ocupe algum lugar nas possíveis posições sociais e seja definido por meio dessas posições (Bourdieu, 1989; 1990; 1996).

Diante da relação entre agentes e estrutura, os campos são campos de força, em que a estrutura exerce força coercitiva sobre os agentes e ao mesmo tempo são campos de lutas, em que os agentes agem conforme suas posições e disposições para ascender na hierarquia social do campo. Assim, qualquer indivíduo que estiver num determinado campo vai agir direcionado por interesses específicos, individuais ou coletivos, de forma a auxiliar na ascensão ou manutenção no sistema de posições no campo (Bourdieu, 1989; 1990; 1996).

Os campos possuem leis gerais e específicas, que determinam a sua existência e os seus limites (Thiry-Cherques, 2006). As leis gerais dos campos são formas de funcionamento que não variam, que podem ser usadas para interpretar diferentes campos, são elas que possibilitam a utilização dos conceitos de Bourdieu em diferentes áreas ou para diferentes referências empíricas, enquanto as leis específicas são particularidades de cada campo.

Dada a compreensão inicial da metáfora do que são os campos nos espaços sociais, compreendemos que o universo de possibilidades de espaços que são considerados campos é tão extenso quanto as possibilidades estilísticas, que fornecem traços distintivos aos indivíduos (Bourdieu, 2007). Os campos são microcosmos, ou o que podemos chamar de mundos, no sentido de mundo artístico, religioso, literário, científico (Thiry-Cherques, 2006).

0 funcionamento de um campo depende da existência de pessoas dispostas a jogar o jogo, implicando no conhecimento e reconhecimento das leis e regras de funcionamento do jogo, isto é, um habitus (Bourdieu, 1983). O habitus faz com que as práticas dos agentes ou de conjuntos de agentes sejam sistemáticas, à medida que são resultados das mesmas aplicações de esquemas de práticas constitutivas de um estilo de vida, sendo, também, sistematicamente diferentes de outras práticas, de outros agentes, pertencentes a outras condições de existência. Com ele, os indivíduos são capazes de diferenciar e apreciar o mundo social (Bourdieu, 2007). Portanto, é um oficio, um capital de técnicas, um conjunto de crenças, e um conjunto de propriedades que se ligam à história do campo, à posição na hierarquia, e que são condições e produtos do funcionamento do campo (Bourdieu, 1983).

O habitus está incorporado, por exemplo, nas ações, nos pensamentos, nos julgamentos do que pode ou não ser feito, dito ou pensado, em modos de ser e, no limite, nos estilos de vida (Bourdieu, 1977). Entretanto isso não significa dizer que todos os indivíduos agem da mesma maneira. As diferenças individuais têm origem nas classes sociais e no momento histórico. As diferenças se formam no conjunto de habitus dos campos que os indivíduos fazem parte, como uma forma de integração entre as disposições de cada campo no decorrer das experiências individuais. As 
experiências iniciais na família, por exemplo, são base para o habitus desenvolvido na escola, que são base para as experiências seguintes do indivíduo, sempre num processo de reestruturação do habitus de acordo com as experiências vividas (Bourdieu, 1977).

Diante disso, classes, no sentido de Bourdieu, são categorias de pessoas que ocupam as mesmas posições no campo, de forma que quanto mais próximas na topologia do campo, maior a chance dos habitus serem compartilhados, constituindo um grupo social. A formação das classes sociais constrói e classifica identidades de grupos que são uma das bases para as lutas nos campos (Jenkins, 2014).

O funcionamento do campo é determinado pela ação dos indivíduos e grupos nas diferentes classes sociais, que são constituídos pelas, e constituem as, relações de força. Os campos são, em sua essência, espaços de lutas simbólicas, entre agentes e/ou instituições, por posicionamento dentro do próprio campo. As lutas são travadas pela definição da cultura legítima e das regras que governam o funcionamento do campo, ou seja, dos princípios de dominação, que fazem com que se mantenham ou sejam alteradas as posições no campo (Bourdieu, 2007).

Os princípios de dominação funcionam como uma economia particular do campo, de forma que os agentes investem e recebem algo em troca de acordo com essa economia (Bourdieu, 1989). Os instrumentos de dominação, que são os princípios de diferenciação, variam com o campo que se observa e são os bens ou recursos chamados de capitais. Existem quatro categorias principais de capitais que Bourdieu analisa: capital econômico, capital simbólico, capital social e capital cultural (Bourdieu, 2007; Jenkins, 2014).

De fato, Bourdieu e Wacquant (2013) esclarecem que existem duas formas em que os indivíduos são socialmente caracterizados. A primeira é por meio de propriedades materiais, que representa o capital econômico e a segunda é por meio de propriedades simbólicas, que são atribuídas aos indivíduos por meio das relações com outros indivíduos que são capazes de perceber essas propriedades de acordo com a lógica social de determinado campo, formando, então, o capital simbólico. Do capital simbólico derivam os outros tipos de capitais, como o social, o cultural, o científico, o literário, entre outros.

A diferença entre o capital econômico e o simbólico é que o capital econômico transparece uma natureza egoísta e instrumental nos indivíduos, enquanto o capital simbólico é representado de forma desinteressada, como se esse tipo de capital fosse buscado pelo bem de todos, como natureza altruísta dos indivíduos. No entanto, esse reconhecimento enganoso representa formas de violência simbólica, o que significa dizer que o capital simbólico é, também, usado como forma de discriminação e hierarquização no sistema social, fonte de vantagens ou desvantagens sociais (Grenfell, 2014).

O capital simbólico é representado pelo prestígio e honra social e só tem significado em determinados contextos em que os indivíduos atribuem valor a propriedades simbólicas específicas. 0 capital social são diversas formas de relações com outros indivíduos significantes no contexto do campo. 0 capital cultural representa algum valor associado com gostos culturais, padrões de consumo, habilidades e premiações. Por exemplo, no campo da educação algum tipo legítimo de conhecimento é considerado capital cultural (Bourdieu, 2007).

Cada campo valoriza cada uma das formas de capital em maior ou menor grau e mudar de um campo para outro pode acarretar em perda de capital (Bourdieu, 2007). Quanto maior o volume e estrutura do capital específico dentro do campo, maior o posicionamento dos indivíduos. Assim, as relações são sempre relações de poder, que dependem do poder material ou simbólico acumulado pelos agentes do campo (Bourdieu, 1983; 1989).

Em complemento, os campos apresentam um movimento de produção e consumo dos agentes e instituições, ambos como formas de manter ou ascender nas posições sociais. Os gostos são manifestados nas práticas (esportes, lazer) e nas propriedades (móveis, roupas, livros), e para que os gostos existam é preciso um sistema de classificação de bens e indivíduos que possuam esses princípios de classificação (Bourdieu, 1983). Há, dessa forma, uma relação entre as lógicas da produção e do consumo. 0 campo de produção fornece aos indivíduos um sistema de possibilidades estilísticas para que eles possam selecionar os bens culturais que se adequam ao seu estilo de vida. Ao mesmo tempo, o campo de produção só existe e funciona porque existem os gostos e propensões a consumir determinados tipos de bens culturais (Bourdieu, 2007). Os agentes especializados de 
produção são direcionados pelos interesses específicos que os mantêm no domínio do campo. Sendo assim, cada campo específico possui agentes de produção simbólica (Bourdieu, 1996), que estão em constante luta por posição.

Diante da relação entre produção e consumo, Bourdieu (2007) argumenta sobre um papel mediador, que auxilia na apresentação e representação da produção cultural perante os consumidores, contribuindo com a formação dos gostos, os intermediários culturais ou taste makers. Uma nova pequena burguesia, considerada uma classe social dotada de gostos e práticas culturais distintas, que envolve ocupações como vendedores, marketing, moda, relações públicas e instituições que oferecem bens e serviços simbólicos. A produção simbólica é, dessa maneira, o centro das ações dos intermediários culturais, que usam de propagandas, marketing e técnicas promocionais para moldar os valores dos bens e, ao mesmo tempo, conectar esses valores com os indivíduos consumidores (Negus, 2002).

Tanto o trabalho da produção cultural, quanto o trabalho dos intermediários culturais influenciam a formação dos gostos dos indivíduos, que, por sua vez, modela as disposições individuais no decorrer do tempo, formando, então, um novo habitus. A mudança nos gostos dos indivíduos de um campo, em última instância, provoca mudanças no campo, podendo influenciar a estrutura, a hierarquia, o capital específico, entre outros elementos, que fazem com que o campo funcione de maneira diferente. Portanto, os jogos dentro dos diferentes campos são sempre jogos em curso e infindáveis, havendo possibilidade de mudanças (Grenfell, 2014).

0 movimento de transformação no campo é sempre vagaroso, pois passa por um processo de adequação entre a história objetivada e a história incorporada, quando essas duas histórias se desviam, uma ou outra é motivada a se adaptar (Bourdieu, 2007; Grenfell, 2014). Dessa maneira, as mudanças de habitus implicam em mudanças no campo, enquanto mudanças no campo geram mudanças no habitus (Bourdieu, 2007). Qualquer que seja a mudança no campo, ela causa uma disjunção entre o habitus e as disposições no campo, trazendo consequências para a formação do campo ao longo do tempo, causando o que Bourdieu denomina como hysteresys. O conceito de hysteresis é concebido por Bourdieu como uma consequência necessária dos conceitos de habitus e campos, isto é, dada a definição de campo e habitus, mudanças em um necessariamente implicam em mudanças no outro.

Assim, revoluções parciais ou totais podem acontecer. As revoluções parciais acontecem a todo o tempo e não colocam em questão o funcionamento do jogo. São pequenas alterações em função das experiências vividas, que mantém o equilíbrio no campo, a estabilidade social (Bourdieu, 1989). Uma vez que o habitus é resistente a mudanças, pois é parte das disposições adquiridas historicamente, o processo de adaptação às pequenas mudanças é longo, lento e muitas vezes inconsciente (Swartz \& Zolberg, 2005). Por outro lado, as revoluções totais, que representam momentos de crises sociais, reformulam o funcionamento do campo, os dominantes e os dominados, o capital em jogo, os habitus dos indivíduos, entre tantos outros elementos que contribuem com o funcionamento do campo (Bourdieu, 1989). Nesses momentos, até que haja a estabilidade novamente no campo, novas oportunidades surgem, gerando consequências para a estrutura do campo ao longo do tempo (Grenfell, 2014).

Essas mudanças nos campos podem resultar em mudanças na hierarquia, no poder e na riqueza do campo, uma vez que os capitais simbólicos e as fontes de legitimação no campo podem ser alterados. Novos campos e subcampos podem surgir e campos podem deixar de existir como resultado das grandes transformações como as citadas por Grenfell (2014).

Os conceitos de Bourdieu não se limitam aos aqui apresentados, mas esses são suficientes para a análise proposta neste trabalho. Diante desses conceitos, ressaltamos que a estrutura dos campos são arenas de produção, circulação e apropriação de bens e serviços, sendo possível e útil analisar os fenômenos de Marketing sob essa perspectiva.

\section{PROCEDIMENTOS METODOLÓGICOS}

Neste estudo de natureza descritiva, realizamos um levantamento da produção acadêmica que está no escopo de Marketing e estudos de consumo e utiliza Bourdieu, parcial ou integralmente, como 
base para a construção da pesquisa. Para tanto, delimitamos um corte transversal temporal de 10 anos (de 2008 até 2018), para analisar os estudos mais recentes.

A pesquisa foi realizada de diversas formas, em busca de encontrar a maior quantidade possível de artigos. Inicialmente, fizemos uma busca nas principais bases de dados (Emerald, Ebsco, Scielo, Spell, Taylor \& Francis, Science Direct, Elsevier, Sage, Capes, Jstor), a fim de coletar artigos de diversas áreas que contemplassem as palavras "Bourdieu" e "marketing" em seu resumo. Posteriormente, a fim de encontrar artigos que fossem da área de marketing e estudos de consumo, mas que não possuíssem o termo "marketing" em seu resumo, pesquisamos diretamente nas bases dos principais jornais acadêmicos da área de Administração, Marketing e estudos de consumo, utilizando somente o termo "Bourdieu" e avaliando se os resultados estavam de acordo com o escopo de marketing, completando a pesquisa. Os jornais pesquisados foram: Journal of Marketing, Journal of Marketing Research, Journal of Marketing Theory and Practice, Journal of International Marketing, Journal of Public Policy \& Marketing, Marketing Science, Journal of Marketing Management, European Journal of Marketing, Journal of the Academy of Marketing Science, Consumption, Markets and Culture, International Journal of Consumer Studies, Journal of Consumer Affairs, Journal of Consumer Behaviour, Journal of Consumer Culture, Journal of Consumer Marketing, Journal of Consumer Policy, Journal of Consumer Research, Revista de Administração de Empresas, Revista de Administração Contemporânea, Revista Brasileira de Gestão de Negócios, Brazilian Administration Review, Brazilian Business Review, Cadernos EBAPE, Revista de Administração Pública, Revista Organizações em Contexto e Revista de Administração da USP.

A literatura encontrada foi acessada e codificada incluindo o nome do periódico em que foi publicada, o título e o ano de publicação, o resumo, os autores, o tema do artigo, os conceitos de Bourdieu que são utilizados no artigo, o enfoque do artigo, se somente teórico ou com dados empíricos, a metodologia utilizada, se qualitativa, quantitativa ou mista, as formas de coleta e análise dos dados e as palavras-chave do artigo.

Para análise dos dados, primeiramente fazemos uma análise com base nas categorias analisadas, a fim de identificar como têm sido feitos os trabalhos com o uso de Bourdieu em Marketing, e posteriormente analisamos os artigos encontrados de forma mais aprofundada, buscando compreender o conhecimento construído no campo e encontrar oportunidades para pesquisas futuras.

\section{RESULTADOS E ANÁLISES Indicadores bibliométricos}

Ao final da coleta foram selecionados 49 artigos para análise. A Tabela 1 apresenta os artigos encontrados classificados por periódico e por ano de publicação.

Tabela 1

Quantidade de publicações por periódico e por ano

\begin{tabular}{|c|c|c|c|c|c|c|c|c|c|c|c|c|}
\hline Ano & 2008 & 2009 & 2010 & 2011 & 2012 & 2013 & 2014 & 42015 & \begin{tabular}{l|l}
52016 \\
\end{tabular} & 2017 & 72018 & Total \\
\hline \multirow{16}{*}{$\begin{array}{l}\text { Brazilian Administration Review } \\
\text { Consumption, Markets and Culture } \\
\text { Critical Perspectives on Accounting } \\
\text { European Journal for Sport and Society } \\
\text { European Journal of Marketing } \\
\text { International Journal of Consumer Studies } \\
\text { Journal of the Academy of Marketing Science } \\
\text { Journal of Consumer Affairs } \\
\text { Journal of Consumer Culture } \\
\text { Journal of Consumer Research } \\
\text { Journal of Marketing Management } \\
\text { Journal of Marketing Research } \\
\text { Journal of Organizational Change Management } \\
\text { Journal of Social Marketing } \\
\text { Management Decision } \\
\text { Marketing Theory }\end{array}$} & & & & & & & & & & & 1 & 1 \\
\hline & & 1 & & & 1 & & & 1 & & 1 & & 4 \\
\hline & & & & & & 1 & & & & & & 1 \\
\hline & & & & & & & 1 & & & & & 1 \\
\hline & & & 1 & 1 & & & 1 & 1 & & & 1 & 5 \\
\hline & & & & & & & 1 & & & & & 1 \\
\hline & & & & & 1 & & & & & & & 1 \\
\hline & & 1 & & & & & & & & & & 1 \\
\hline & & 1 & & 1 & & 4 & 2 & & 2 & 2 & & 12 \\
\hline & & & 1 & & 1 & 1 & 1 & 1 & & & & 5 \\
\hline & & & & 1 & & & & & 1 & & & 2 \\
\hline & & & & & & & & & 1 & & & 1 \\
\hline & & & & & & & & & 1 & & & 1 \\
\hline & & & & & & 1 & 1 & & & & & 2 \\
\hline & & & & & & 1 & & & & & & 1 \\
\hline & 1 & & & & & & & & & & & 1 \\
\hline
\end{tabular}


Qualitative Market Research

Recherche et Applications en Marketing

Revista de Administração de Empresas

Revista de Negócios

Revista Organizações em Contexto

Revista Pensamento Contemporâneo em

Administração

Social Semiotics

Sport in Society

The Australian Educational Researcher Quantidade de artigos

Fonte: Elaborada pelos autores.

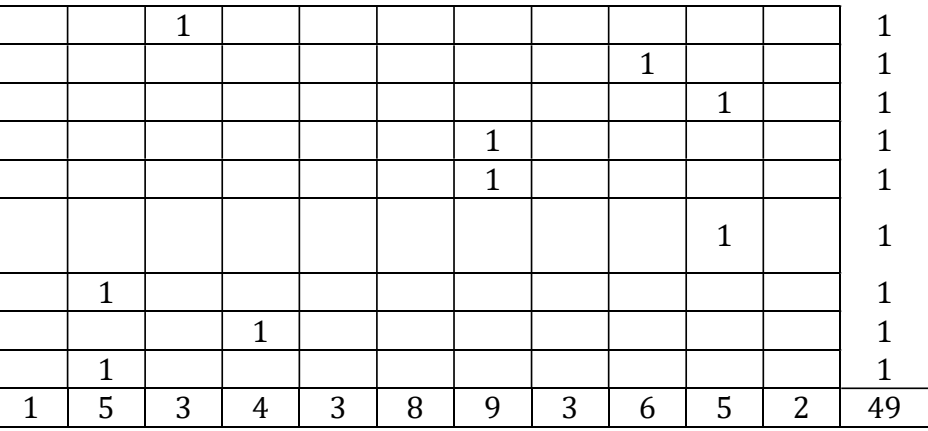

Podemos perceber que há predomínio de publicações em revistas com ênfase em consumo, representando 47\% (23 artigos) do total. Isso pode ser explicado pelo surgimento e consolidação da corrente denominada CCT, que passou a ser utilizada nas pesquisas de consumo, como um manifesto (Bode \& Ostergaard, 2013) contra o mainstream positivista e racional que se estabelecia, que desconsiderava aspectos do consumo como as emoções, as relações e os significados embutidos nas decisões dos consumidores. Esse processo se inicia por volta dos anos 80 (Arnould \& Thompson, 2007; Bode \& Ostergaard, 2013) e se intensifica ao longo do tempo, integrando um conjunto de pesquisas que se moviam numa mesma direção.

Tais circunstâncias, segundo Thompson, Arnould e Giesler (2013), fez com que novas perspectivas teóricas fossem adotadas pelos pesquisadores de CCT. A partir disso diversos renomados estudiosos da sociologia passaram a ser considerados nos estudos da CCT, como Theodor Adorno, Pierre Bourdieu, Michel Foucault, entre outros (Thompson, Arnould \& Giesler, 2013).

Em complemento, nos artigos analisados, podemos perceber uma predominância do uso de alguns conceitos de Bourdieu, conforme Tabela 2. Foram selecionados os conceitos base para a estruturação de cada trabalho, isto é, aqueles que tinham predominância.

Tabela 2

Uso dos conceitos de Pierre Bourdieu

\begin{tabular}{lc}
\hline \multicolumn{1}{c}{ Conceito } & Quantidade de trabalhos \\
\hline Capital & 21 \\
Habitus & 15 \\
Gosto & 11 \\
Campos & 10 \\
Classe social & 6 \\
Teoria da prática & 5 \\
Intermediários Culturais & 4 \\
Distinção & 4 \\
Violência simbólica & 2 \\
Hierarquia & 2 \\
Agência & 2 \\
Produção cultural/ simbólica & 2 \\
Doxa & 1 \\
Dominação simbólica & 1 \\
Violência simbólica & 1 \\
Reprodução social & 1 \\
Hysteresis & 1 \\
\hline
\end{tabular}

Fonte: Elaborada pelos autores.

Diante disso podemos perceber que há um uso comum dos conceitos de capital, habitus, campos e gosto. Isso pode ser explicado por serem conceitos que formam a base da literatura de Bourdieu. No entanto, destacamos que alguns conceitos amplamente relacionados com os interesses 
de marketing nos espaços sociais, como os conceitos de produção simbólica, intermediários culturais, reprodução social e hysteresis, são pouco usados.

Em relação à metodologia, dos trabalhos analisados 5 (10\%) são teóricos, 40 (82\%) são qualitativos, 3 (6\%) são quantitativos e 1 (2\%) é misto. As Tabelas 3 e 4 em sequência apresentam os métodos de coleta e análise de dados, respectivamente, nos artigos coletados. É importante ressaltar que diversos trabalhos utilizam mais de uma forma de coleta e análise dos dados, de forma que o total de métodos não corresponde ao total de trabalhos analisados.

Tabela 3

Métodos de coleta dos dados

\begin{tabular}{lc}
\hline \multicolumn{1}{c}{ Métodos } & Quantidade de trabalhos \\
\hline Entrevista & 35 \\
Observação & 13 \\
Dados secundários & 12 \\
Grupo de foco & 6 \\
Estudo de caso & 2 \\
Survey & 2 \\
Painél & 1 \\
Delphi & 1 \\
\hline
\end{tabular}

Fonte: Elaborada pelos autores.

Destacamos que as observações aconteceram em diversos níveis, sendo a mais comum a observação participante. Em relação aos dados secundários, foram utilizados documentos, fotos, propagandas, livros, jornais e revistas. Diante disso, podemos perceber que é muito comum o uso de entrevistas, dados secundários e observação para a compreensão dos fenômenos por meio dos conceitos de Bourdieu. É possível, ainda, perceber que outros métodos, embora pouco utilizados, são capazes de auxiliar na explicação de fenômenos em conjunto com os conceitos de Bourdieu.

Tabela 4

Métodos de análise dos dados

\begin{tabular}{lc}
\hline \multicolumn{1}{c}{ Métodos } & Quantidade de trabalhos \\
\hline Análise interpretativa com & base \\
nos conceitos de Bourdieu & 12 \\
Análise de discurso & 9 \\
Hermenêutica & 7 \\
Grounded Theory & 6 \\
Análises estatísticas & 4 \\
Análise comparativa & 3 \\
Análise categórica indutiva & 1 \\
Análise de conteúdo & 1 \\
Semiótica & 1 \\
\hline
\end{tabular}

Fonte: Elaborada pelos autores.

Sobre a análise de dados, notamos que grande parte dos trabalhos não apresenta explicitamente a forma de análise utilizada. Em alguns trabalhos conseguimos especificar que tipo de análise foi feita, no entanto em 5 trabalhos não foi possível identificar a forma de análise. Apesar disso, percebemos que muitos trabalhos utilizam análises interpretativas com base em conceitos de Bourdieu. Por outro lado, ressaltamos que a análise de discurso, a grounded theory e a análise hermenêutica são bastante utilizadas para interpretar os fenômenos tendo como referência os trabalhos de Bourdieu.

Mills e Gale (2007) informam que pesquisadores que utilizem a perspectiva de Bourdieu para guiar sua análise devem compreender a dinâmica de interação entre os indivíduos e os ambientes que os cercam, situando-os no contexto analisado. Além disso, Bourdieu (1989) considera que a linguagem 
dos agentes pode ser determinada e direcionada pelo seu habitus e pela própria condição no campo, tanto como forma de posicionamento, quanto como forma de luta.

Dessa maneira, a análise de discurso parece ter destaque por compartilhar de diversos pressupostos com Bourdieu (Bourdieu, 1989; Gill, 2000), como: uma visão crítica em relação ao conhecimento pronto, dado; a compreensão da construção social dos indivíduos; a compreensão de que a construção dos discursos é alinhada aos contextos vivenciados pelos indivíduos; a visão da vida social compreendendo conflitos de diversas naturezas, sendo que o discurso é usado como forma de posicionamento diante desses conflitos.

Ademais, a Figura 1 demonstra as palavras-chave dos artigos analisados. Destacamos o uso de palavras como consumo político, ético e sustentável, bem como questões de gênero e etnicidade, como amplamente representantes dos estudos analisados, em conjunto com os principais conceitos de Bourdieu. Além disso, questões de alimentação, vestuário, educação e identidade do consumidor tiveram destaque.

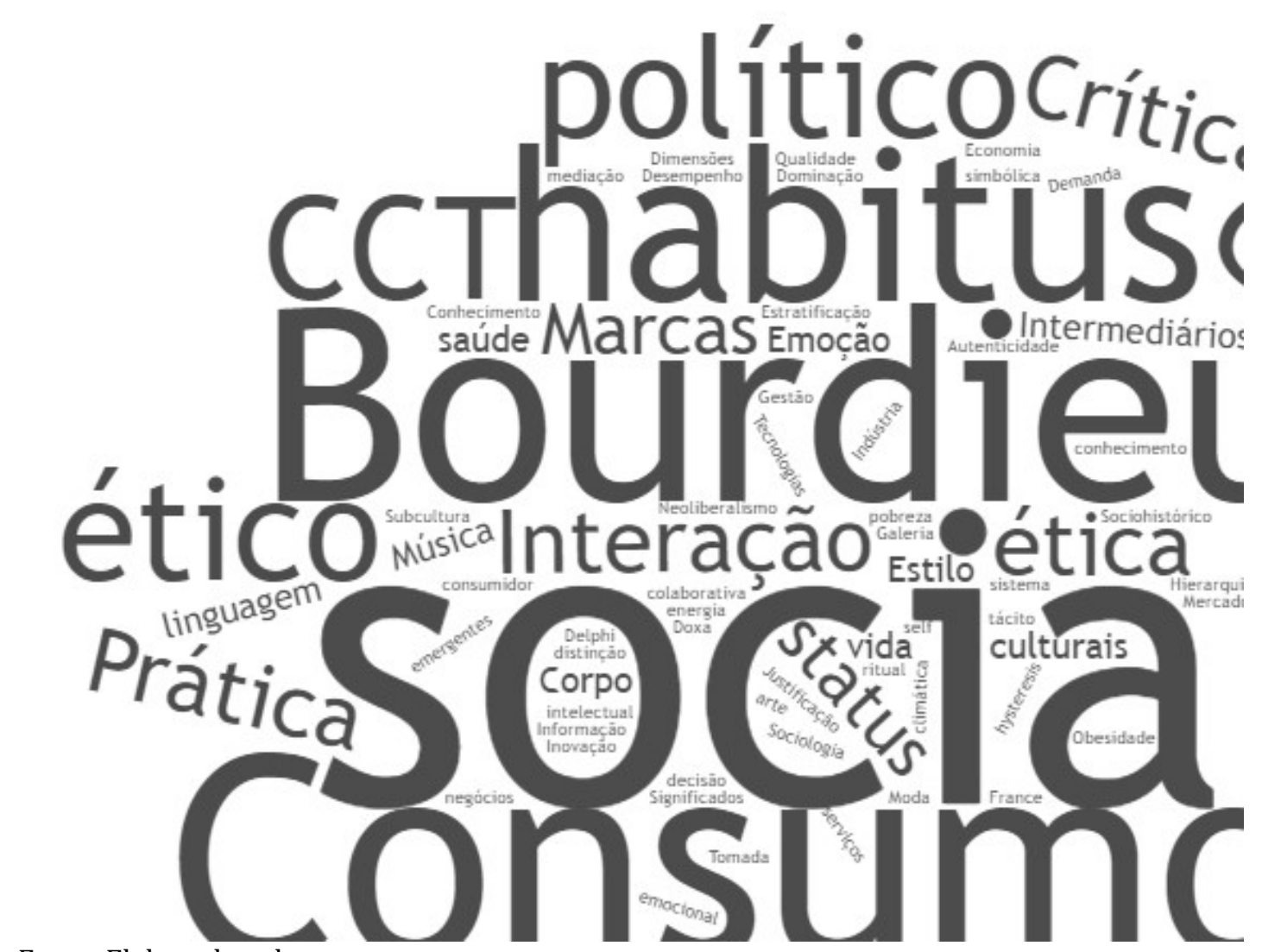

Fonte: Elaborada pelos autores.

Figura 1: Nuvem de palavras-chave dos artigos analisados

Antes de realizamos análises e conclusões mais aprofundadas sobre os resultados encontrados, na próxima seção analisamos os principais temas de interesse nos artigos coletados.

\section{Pierre Bourdieu em Marketing e Estudos de Consumo}

Primeiramente, podemos notar que os trabalhos analisados possuem origem e interesse em diversas áreas. Por exemplo, Adkins e Corus (2009) analisam a formação do conhecimento sobre saúde como prática social e cultural, de forma que aumentar o conhecimento dos indivíduos sobre saúde pode ajudar a resolver problemas de saúde pública. Os autores usam a teoria da prática para mostrar as estratégias dos mercados de saúde para desenvolver essas formas de capital nos 
indivíduos. Envolvendo a educação, English (2009) analisa que famílias de subúrbios na Austrália preferem escolas particulares às governamentais devido ao capital cultural que elas podem proporcionar aos seus filhos, criando um significado de uma vida melhor para eles. Ainda, Nascimento, Costa Filho e Hor-Meyll (2017) investigam os significados atribuídos à educação privada por famílias pertencentes à nova classe média brasileira, mostrando que a escolha da educação privada está relacionada ao processo de ascensão social de um determinado segmento da população.

Atencio e Beal (2011) investigam como artes e esportes são usados como forma de legitimação de discursos e disposições no campo social. Ademais, Ferry (2014) apresenta porque as escolas passaram a incentivar práticas esportivas e porque os esportes são tão viáveis para as escolas numa visão de mercado, sob o argumento de que os esportes produzem formas de capital para as escolas devido aos vários significados associados a eles. Em complemento, temas sociais como corrupção e comportamento feminino são estudados por Vaidya (2019) e Sinclair (2017), respectivamente, mostrando o alcance das teorias de Bourdieu para os estudos de diversas áreas.

No contexto de marketing e estudos de consumo muitas pesquisas são realizadas, isso porque as teorias de Bourdieu são usadas principalmente para compreensão de fenômenos culturais e sociais. Encontram-se publicações sobre gestão (Lowe et al., 2016), propaganda (Chavez, 2012; Drumwright \& Kamal, 2016; Kaptan, 2013; Kobayashi, 2017), qualidade (Imrie, 2013), marcas (Leão, Mello \& Gaião, 2014) e diferentes tipos de mercados (Brei e Tadajewski, 2015).

Sobre gestão, Lowe, Rod, Kainzbauer et al. (2016) exploram como diferentes relacionamentos são caracterizados entre os agentes de mercado para determinar se as teorias sociais da prática, como a de Bourdieu, são entendidas como práticas para os gestores. No âmbito de propagandas, Chavez (2012) visualiza a indústria da propaganda como um campo, analisando a relação entre capital econômico e cultural dos praticantes em busca de distinção nos mercados. Drumwright e Kamal (2016) investigam como o discurso das propagandas influencia o habitus e doxa do campo das propagandas e como esses intermediários culturais enfrentam questões éticas. Kobayashi (2017) estuda como o processo de trabalho criativo e cultural atravessa os campos de produção simbólica e consumo por meio dos quais a cultura popular e os gostos são negociados.

Ao falar de qualidade, Imrie (2013) busca compreender a maneira pela qual as interações sociais influenciam a avaliação da qualidade dos serviços. Sobre marcas, Leão, Mello e Gaião (2014) avaliam como os consumidores significam as marcas, num processo de distinção social, especialmente em relação a status social, estilo de vida e diferenças pessoais. Brei e Tadajewski (2015) por sua vez utilizam o referencial de Bourdieu para analisar o contexto histórico e cultural do crescimento do mercado de água engarrafada no mercado Francês. Ainda no contexto de Marketing, encontramos a pesquisa de Farjaudon e Morales (2013), que observa as lutas de poder no campo da contabilidade e marketing. Utilizando conceitos de dominação simbólica e poder simbólico, os autores argumentam que esses campos competem pelo monopólio da definição de valor e pela habilidade de representar as organizações.

Quando se fala em consumo, comportamento do consumidor e cultura de consumo, uma vasta aplicação dos conceitos Bourdieusianos é encontrada. Existem trabalhos que tratam de consumo de alimentos e bebidas (Costa, Zepeda, \& Sirieix, 2014; Crivits \& Paredis, 2013; Jarvinen, Ellergaard, \& Larsen, 2014; Quintão, Brito, \& Belk, 2017), esportes (Thompson e Usturner, 2015), religião (Mcalexander et al., 2014) e roupas (Tsaousi, 2016), entre outros. Jarvinen, Ellergaard e Larsen (2014) associam o consumo de álcool com os gostos e status social dos indivíduos. Costa, Zepeda e Sirieix (2014) investigam o uso de alimentos orgânicos como símbolos de status social. Crivits e Paredis (2013) analisam a transição das práticas de consumo alimentar para uma abordagem sustentável. Quintão, Brito e Belk (2017) avaliam os rituais de consumo de cafés especiais e a transformação dos gostos dos consumidores. Para os autores há um ritual de transformação do gosto, em que os consumidores passam a diferenciar o consumo anterior, de massa, e o consumo atual. McAlexander, Dufault, Martin et al. (2014) estudam as consequências nos indivíduos das alterações em instituições que os formam, como as igrejas. Tsaousi (2014) explora o consumo de roupas íntimas femininas como forma de construção da identidade.

De modo complementar, alguns trabalhos avaliam o consumo por si só. Herbert e CollinLachaud (2016) analisam os mecanismos transformativos do consumo colaborativo, utilizando os 
conceitos de habitus e hysteresis. Saatcioglu e Ozanne (2013) exploram como disposições morais diferentes formam as práticas de consumo e negociações dos indivíduos de baixa renda, por meio do conceito de capital cultural. Usturner e Holt (2009) com conceitos de campos, capital cultural e habitus investigam como o consumo por status é realizado nas classes médias de países menos industrializados. Ponte e Campos (2018) descrevem a transformação de gosto no processo de mobilidade social. As autoras propõem o estudo das transformações de gosto por meio de dois aspectos: a dinâmica da distinção durante a mobilidade e a aquisição de gostos de forma inercial, sem intenção. Além dessas, outras pesquisas avaliam hábitos de consumo, comportamentos de consumo e diferenças socioculturais de consumo, entre outros aspectos (ex.: Usturner \& Thompson, 2010; Halnon \& Cohen, 2006).

Ademais, existem estudos que avaliam questões metodológicas sobre as teorias de Bourdieu, como a aplicabilidade das abordagens teóricas e práticas de Bourdieu nos estudos de Marketing e consumo (El-amir \& Burt, 2010; Roling \& Vieira, 2014; Tapp \& Waren, 2010). Esses estudos evidenciam a interdisciplinaridade dos conceitos de Bourdieu e sua ampla aplicabilidade para explicar as construções que envolvem o comportamento dos indivíduos e instituições presentes nos mercados.

Como pode ser percebido nos estudos analisados, os conceitos de Bourdieu são utilizados para compreender fenômenos sociais e culturais e por esse motivo podem ser amplamente utilizados para compreensão de fenômenos de Marketing e estudos de consumo. Essa abordagem permite observar as relações de poder que se estabelecem em cada campo ao longo do tempo, auxiliando na compreensão de conflitos e comportamentos dos agentes.

\section{DISCUSSÃO E AGENDA DE PESQUISA}

Diante dos trabalhos apresentados, desenvolvemos uma agenda de pesquisa para propor ampliar a utilização da literatura de Bourdieu nos estudos de Marketing e consumo. Acreditamos que esse tipo de abordagem pode enriquecer os trabalhos, apresentar novas perspectivas sobre fenômenos já observados e incentivar novos insights, uma vez que identificamos os conceitos utilizados por Bourdieu para a compreensão dos espaços sociais como presentes e formadores das relações entre produção e consumo, estando no escopo de Marketing e de consumo.

Para desenvolver a agenda de pesquisa, utilizamos os padrões identificados nas pesquisas já existentes e com base neles realizamos uma discussão e apresentamos possibilidades de pesquisas em Marketing e estudos de consumo que utilizem os conceitos de Bourdieu, conforme Tabela 5. 

Tabela 5

Agenda de pesquisa

\begin{tabular}{|c|c|c|c|}
\hline Padrões & Discussão & Agenda de pesquisa & Exemplos \\
\hline $\begin{array}{l}\text { Ênfase em } \\
\text { estudos de } \\
\text { consumo }\end{array}$ & $\begin{array}{l}\text { Atribuímos a grande participação de } \\
\text { estudos de consumo à abordagem de CCT, } \\
\text { que considera a utilização de teorias } \\
\text { sociológicas e antropológicas para uma } \\
\text { compreensão menos racional e utilitária } \\
\text { dos fenômenos. No entanto, consideramos } \\
\text { que outras áreas dos estudos de Marketing } \\
\text { também podem se beneficiar por essa } \\
\text { visão, uma vez que todas as práticas de } \\
\text { marketing acontecem em espaços sociais. }\end{array}$ & $\begin{array}{l}\text { Propomos continuar com pesquisas envolvendo } \\
\text { consumidores, especialmente comportamentos de consumo e } \\
\text { seus significados, comparação entre discursos e práticas de } \\
\text { agentes de consumo, consumo como forma de legitimação de } \\
\text { discursos e ascensão em espaços sociais. } \\
\text { Também propomos pesquisas que ampliem o escopo de } \\
\text { Marketing como pesquisas sobre estratégias de crescimento e } \\
\text { legitimação de mercados, estratégias de agentes de mercado } \\
\text { para a obtenção dos diferentes tipos de capital, relações entre } \\
\text { capitais em diferentes mercados, mudanças de mercado e } \\
\text { perdas de capital. }\end{array}$ & $\begin{array}{l}\text { Como o consumo de produtos nocivos à } \\
\text { saúde permanece por meio dos significados } \\
\text { associados a eles? Qual a relação entre o } \\
\text { discurso e a prática de sustentabilidade dos } \\
\text { agentes produtores? De que forma o } \\
\text { consumo de alimentos é utilizado para } \\
\text { legitimar discursos? Como a religião pode } \\
\text { contribuir para a legitimação de mercados? } \\
\text { Quais as estratégias dos youtubers para } \\
\text { alcançar capital social? Como acontece a } \\
\text { perda de capital na mudança do mercado de } \\
\text { professores para o mercado de consultoria? }\end{array}$ \\
\hline
\end{tabular}

Além de continuar com pesquisas que visualizem os espaços sociais de atuação de Marketing como campos, analisando os habitus dos agentes e os capitais em jogo, propomos ampliar a utilização dos conceitos de Bourdieu. Por exemplo, o conceito de intermediários culturais ainda é pouco explorado na literatura. Podem ser feitos estudos que analisem a relação entre esses agentes com os produtores para compreender a Como dito no referencial teórico, todos os temporalidade do desenvolvimento de intermediários Ênfase nos conceitos de Bourdieu convergem para a conceitos de noção de campos, em que o habitus, o campos, gosto e os capitais são os principais capital, elementos que caracterizam um campo. habitus e No entanto, diversos conceitos de gosto Bourdieu podem enriquecer os estudos de Marketing.

culturais, bem como a sua forma de legitimação diante de mercados, ou até mesmo intermediários culturais que exercem influências negativas ou que são rejeitados pelos consumidores, de forma que estes passem a ressignificar práticas ao invés de reproduzi-las. Um outro conceito que pode ser amplamente usado nas pesquisas em Marketing é o de hysteresis, que auxilia na compreensão da transformação de campos, ou seja, de mercados. Além disso, outros conceitos como poder simbólico, dominação simbólica, produção simbólica e reprodução cultural podem ser explorados para a compreensão de fenômenos de Marketing.

Como é a relação entre jornalistas e marcas na formação da publicidade? Qual o "ciclo de vida" de um intermediário cultural? Quais os fatores que influenciam essa temporalidade?

Como intermediários culturais rejeitados pelos consumidores contribuem para a ressignificação de práticas de consumo?

Quais estratégias de mercados são utilizadas para legitimar formas de consumo prejudiciais à saúde dos consumidores?

\begin{tabular}{|c|c|c|c|c|c|c|}
\hline $\begin{array}{l}\text { Ênfase nas } \\
\text { formas de }\end{array}$ & $\begin{array}{l}\text { Embora tenha predominância } \\
\text { entrevistas, observação e }\end{array}$ & $\begin{array}{r}\text { de } \\
\text { dados }\end{array}$ & $\begin{array}{l}\text { Propomos a realização de pesquisas com diferentes métodos } \\
\text { de coleta de dados, envolvendo dados qualitativos (corpus), }\end{array}$ & $\begin{array}{l}\text { Por exemplo, } \\
\text { demográficos: }\end{array}$ & dados & quantitativos \\
\hline
\end{tabular}

Consumer Behavior Review, 4(3), 199-216 
coleta de

dados

secundários, outras técnicas vêm sendo utilizadas, mostrando o potencial do referencial de Bourdieu para diversos tipos de pesquisa. É interessante notar que Bourdieu constrói seu referencial teórico sem amarras epistemológicas, embora se situe no que ele chama de estruturalismo genético. Isso permite certa flexibilidade no uso dos conceitos de Bourdieu por diversos olhares, de forma que o mais importante é romper com o conhecimento pré-existente para conseguir apreender a realidade social (Bourdieu, 1989).

Assim como na coleta de dados, a análise mostrou algumas predominâncias como as análises interpretativas com base nas $\begin{array}{lll}\text { Ênfase na } & \text { categorias de Bourdieu, análise de } \\ \text { discurso e análise hermenêutica. No }\end{array}$ análise de dados entanto, visualizamos possibilidades de diferentes tipos de análise, o que corrobora o afirmado anteriormente sobre as possibilidades metodológicas da utilização dos conceitos de Bourdieu.

Ênfase em São normalmente questões que envolvem temas algum tipo de luta simbólica ou dominação simbólica nos campos. Esses temas, políticos, propriamente de cunho social, são muito políticos, como ética, sustentabilid

ade, questões de

etnia e

gênero como é na maioria dos estudos analisados, e também dados quantitativos. Os dados quantitativos demográficos podem identificar relações entre formas de consumo e significados relacionados a classes sociais, determinar padrões de gostos e estilos de vida de diferentes campos ou segmentos de campos, apreender a valorização dos diferentes tipos de capital por agentes de um determinado campo, entre outros.

Revelam relações entre formas de consumo e significados relacionados a classes sociais;

Determinam padrões de gostos e estilos de vida de diferentes campos;

Apreendem a valorização dos diferentes tipos de capital por agentes de um determinado campo, entre outros.

Propomos uma maior utilização de análises estatísticas descritivas, ou até mesmo multivariadas, como feito por Stamer (2016) em conjunto com análises qualitativas. Por exemplo, os trabalhos que analisam gosto, habitus e estilos de vida podem se beneficiar de análises estatísticas que encontrem padrões de consumo.

\section{(a)}

Prot

Propomos continuar e ampliar as pesquisas sobre questões sociais e políticas, que buscam revelar lutas simbólicas e práticas de diferentes agentes em busca de posicionamento nos campos, ou estratégias de mercados para legitimar formas de consumo prejudiciais à saúde física e/ou mental dos consumidores. Por outro lado, propomos utilizar diferentes e diversos temas que incorporem espaços de atuação de marketing em que as lutas não são tão explícitas, mas acontecem.
Os trabalhos que analisam gosto, habitus e estilos de vida podem se beneficiar de análises estatísticas que encontrem padrões de consumo.

\section{Nota. Fonte: Elaborada pelos autores.}

Quais as estratégias para aquisição de diferentes tipos de capital em ambientes de trabalho? Quais capitais dos agentes de vendas que auxiliam na criação de valor para os consumidores? Como acontecem as transformações de mercados por meio de revoluções, como tecnológicas ou educacionais? Quais as práticas de distinção entre empresas que atuam num mesmo mercado? 
Acreditamos que pesquisas nas direções apresentadas podem enriquecer a literatura de Marketing e estudos de consumo tanto analisando fenômenos por novas perspectivas, quanto ampliando suas possibilidades de interpretação.

\section{CONSIDERAÇÕES FINAIS}

Neste estudo propomos analisar o estado da arte em Marketing e estudos de consumo na utilização dos conceitos de Bourdieu para desenvolver uma agenda de pesquisa para os próximos anos. Os principais resultados dos artigos analisados apontam para uma ênfase em estudos relacionados ao consumo; ênfase em alguns conceitos principais de Bourdieu, como habitus, campo, capital e gostos; ênfase em metodologias qualitativas, com diferentes métodos de coleta e análise de dados, embora haja predominância de entrevistas, observação e dados secundários para a coleta, bem como análise interpretativa, hermenêutica e de discurso para a análise dos dados; prioridade para temas sociais e políticos, que envolvem ética e comportamento sustentável.

Com base nesses resultados, traçamos uma agenda para pesquisas em Marketing e estudos de consumo, considerando as diversas possibilidades que o referencial teórico e metodológico de Bourdieu proporciona. Ressaltamos que as possibilidades apresentadas aqui não se esgotam, são apenas uma tentativa de direcionar os próximos estudos, com o intuito de ampliar a utilização dessa abordagem em Marketing e estudos de consumo. Com isso, esperamos contribuir com o campo ao direcionar novas pesquisas, incentivar novos olhares e dar sustentação para a utilização deste arcabouço teórico.

Pesquisas em Marketing e estudos de consumo que utilizem os conceitos de Bourdieu podem gerar diversas implicações para a prática Administrativa. Primeiro, os conceitos e teorias de Bourdieu circundam ideias que correspondem ao escopo de Marketing e de estudos de consumo. Por exemplo, Bourdieu teoria e esclarece sobre relações entre produção e consumo de bens e serviços, sobre produção simbólica e reprodução cultural de significados de bens e serviços, sobre práticas em busca de legitimação nos mercados, sobre lutas de poder em campos específicos, entre outros diversos temas que se relacionam com o trabalho de Marketing e estudos de consumo. Dessa maneira, analisar problemas e preocupações de Marketing estudos de consumo sob a perspectiva de Bourdieu, pode auxiliar na busca de explicações e soluções tanto do ponto de vista teórico, quanto prático.

Segundo, por corresponder a uma perspectiva sociológica e antropológica, os conceitos de Bourdieu são amplamente úteis para a compreensão de relações entre agentes de cada campo, revelando explicações para comportamentos, preferências, gostos, padrões, entre outras coisas. Entender relações entre consumidores, produtores, produtos, intermediários culturais, nas diversas camadas da sociedade, pode favorecer a prática administrativa direcionando-a para as melhores ações sobre cada uma das relações existentes.

Terceiro, os pesquisadores e as organizações podem utilizar os conceitos de Boudieu para se posicionar diante do campo em que atuam e compreender o seu papel como pertencentes na luta, sendo uns formadores de opinião, outros dominados ou dominantes no campo, e assim, ressaltar cada vez mais questões éticas, políticas, sustentáveis e de direitos humanos, mesmo que como forma de se promover e manter ou alterar sua posição no campo, mas de qualquer maneira pensando no bemestar social.

Como limitações destacamos o uso de revistas escolhidas pelos pesquisadores, podendo haver outras revistas com pesquisas de Marketing e estudos de consumo que utilizem Bourdieu como referência. Também, informamos que alguns artigos não apresentaram palavras-chave, deixando de fazer parte desta análise.

Como sugestões para pesquisas futuras indicamos a necessidade de revisões assim como esta de tempos em tempos, de forma que os trabalhos acumulados ao longo do tempo possam ser sistematizados e novas possibilidades de pesquisas possam ser traçadas. Sugerimos ainda, pesquisas que investiguem a utilização dos conceitos de Bourdieu em Marketing e estudos de consumo, ou até mesmo que desenvolvam novas formas de aplicação desses conceitos. 


\section{Referências}

Adkins, N., \& Corus, C. (2009). Health literacy for improved health outcomes: effective capital in the marketplace. Journal of Consumer Affairs, 43(2), 199-222.

Anderson, P. F. (1983). Marketing, scientific progress, and scientific method. Journal of marketing, $47(4), 18-31$.

Arnould, E., \& Thompson, C. (2007). Consumer culture theory (and we really mean theoretics): dilemmas and opportunities posed by an academic branding strategy. Research in Consumer Behavior, 11, 3-22.

Askegaard, S., \& Linnet, J. T. (2011). Towards an epistemology of consumer culture theory: Phenomenology and the context of context. Marketing Theory, 11(4), 381-404.

Atencio, M., \& Beal, B. (2011). Beautiful losers: the symbolic exhibition and legitimization of outsider masculinity. Sport in Society, 14(1), 1-16.

Bode, M., \& Østergaard, P. (2013). 'The wild and wacky worlds of consumer oddballs' Analyzing the manifestary context of consumer culture theory. Marketing Theory, 13(2), 175-192.

Bonfim, I. D. O. B. (2013). Uma análise da Copa das Confederações de Futebol da FIFA 2013 sob a luz da Teoria dos Campos de Pierre Bourdieu. PODIUM Sport, Leisure and Tourism Review, 2(2), 76-94.

Bourdieu, P. (1977). Outline of a Theory of Practice (Vol. 16). Cambridge university press.

Bourdieu, P. (1983). Questões de sociologia. Rio de Janeiro: Marco Zero.

Bourdieu, P. (1989). O poder simbólico. Rio de Janeiro: Bertrand.

Bourdieu, P. (1990). Coisas ditas. São Paulo: Editora Brasiliense.

Bourdieu, P. (1996). Razões práticas: sobre a teoria da ação. São Paulo: Papirus Editora.

Bourdieu, P. (2007). A distinção crítica social do julgamento. São Paulo: Edusp.

Bourdieu, P., Chamboredon, J. C., \& Passeron, J. C. (1999). A profissão de sociólogo: preliminares epistemológicas. Petrópolis: Vozes.

Bourdieu, P., \& Wacquant, L. (2013). Symbolic capital and social classes. Journal of Classical Sociology, 13(2), 292-302.

Brei, V., \& Tadajewski, M. (2015). Crafting the market for bottled water: a social praxeology approach. European Journal of Marketing, 49(3/4), 327-349.

Chávez, C. (2012). Hispanic agencies and profits of distinction: An examination of the advertising industry as a field of cultural production. Consumption Markets \& Culture, 15(3), 307-325.

Collyer, F. M., Willis, K. F., \& Lewis, S. (2017). Gatekeepers in the healthcare sector: Knowledge and Bourdieu's concept of field. Social Science \& Medicine, 186, 96-103.

Costa, S., Zepeda, L., \& Sirieix, L. (2014). Exploring the social value of organic food. International Journal of Consumer Studies, 38(3), 228-237.

Crivits, M., \& Paredis, E. (2013). Designing an explanatory practice framework: Local food systems as a case. Journal of Consumer Culture, 13(3), 306-336.

Drumwright, M. E., \& Kamal, S. (2016). Habitus, doxa, and ethics: insights from advertising in emerging markets in the Middle East and North Africa. Consumption Markets \& Culture, 19(2), 172-205.

El-Amir, A., \& Burt, S. (2010). Modeling in branding: a critical ethnography approach. Qualitative Market Research: An International Journal, 13(2), 189-209.

English, R. (2009). Selling education through "culture": responses to the market by new, nongovernment schools. The Australian Educational Researcher, 36(1), 89-104.

Farjaudon, A. L., \& Morales, J. (2013). In search of consensus: The role of accounting in the definition and reproduction of dominant interests. Critical Perspectives on Accounting, 24(2), 154-171.

Ferry, M. (2014). School sports is the solution: What is the problem? European Journal for Sport and Society, 11(4), 353-369.

Gill, R. (2000). Discourse analysis (pp. 172-190). Sage Publications.

Grenfell, M. J. (Ed.). (2014). Pierre Bourdieu: key concepts. UK: Routledge.

Halnon, K. B., \& Cohen, S. (2006). Muscles, motorcycles and tattoos: Gentrification in a new frontier. Journal of Consumer Culture, 6(1), 33-56. 
Herbert, M., \& Collin-Lachaud, I. (2017). Collaborative practices and consumerist habitus: An analysis of the transformative mechanisms of collaborative consumption. Recherche et Applications en Marketing, 32(1), 40-60.

Holliday, R., \& Cairnie, A. (2007). Man made plastic: Investigating men's consumption of aesthetic surgery. Journal of Consumer Culture, 7(1), 57-78.

Imrie, B. C. (2013). The influence of social capital on service quality evaluation. Management Decision, 51(4), 871-889.

Järvinen, M., Ellergaard, C. H., \& Larsen, A. G. (2014). Drinking successfully: alcohol consumption, taste and social status. Journal of Consumer Culture, 14(3), 384-405.

Jenkins, R. (2014). Pierre bourdieu. Routledge.

Kaptan, Y. (2013). 'We just know!': Tacit knowledge and knowledge production in the Turkish advertising industry. Journal of Consumer Culture, 13(3), 264-282.

Kitchin, P. J., \& Howe, P. D. (2013). How can the social theory of Pierre Bourdieu assist sport management research?. Sport Management Review, 16(2), 123-134.

Kobayashi, K., Jackson, S. J., \& Sam, M. P. (2018). Multiple dimensions of mediation within transnational advertising production: cultural intermediaries as shapers of emerging cultural capital. Consumption Markets \& Culture, 21(2), 129-146.

Lavie, N., \& Dhoest, A. (2015). 'Quality television'in the making: The cases of Flanders and Israel. Poetics, 52, 64-74.

Leão, A. L. M. S., de Mello, S. C. B., \& da Silva Gaião, B. F. (2014). Uso das marcas na vida cotidiana como busca por distinção social. Revista Organizações em Contexto, 10(20), 85-116.

Lowe, S., Rod, M., Kainzbauer, A., \& Hwang, K. S. (2016). Exploring the perceived value of social practice theories for business-to-business marketing managers. Journal of Organizational Change Management, 29(5), 751-768.

McAlexander, J. H., Dufault, B. L., Martin, D. M., \& Schouten, J. W. (2014). The marketization of religion: Field, capital, and consumer identity. Journal of Consumer Research, 41(3), 858-875.

McCandless, T. (2015). Classing schools. Discourse: Studies in the Cultural Politics of Education, 36(6), 808-820.

Mills, C., \& Gale, T. (2007). Researching social inequalities in education: Towards a Bourdieuian methodology. International Journal of Qualitative Studies in Education, 20(4), 433-447.

Naidoo, R., Shankar, A., \& Veer, E. (2011). The consumerist turn in higher education: Policy aspirations and outcomes. Journal of Marketing Management, 27(11-12), 1142-1162.

Nascimento, E. S., da Costa Filho, M. C. M., \& Hor-Meyll, L. F. (2017). Significados da educação privada na nova classe média. Revista Pensamento Contemporâneo em Administração, 11(2), 32-46.

Natt, E. D. M., \& Ichikawa, E. Y. (2013). O Simbólico em Construções: Estudando a Vila Barrageira da UHE Engenheiro Sérgio Motta à Luz de Pierre Bourdieu. Revista de Ciências da Administração, 15(36), 159-173.

Negus, K. (2002). The work of cultural intermediaries and the enduring distance between production and consumption. Cultural Studies, 16(4), 501-515.

Neu, D., Everett, J., Rahaman, A. S., \& Martinez, D. (2013). Accounting and networks of corruption. Accounting, Organizations and Society, 38(6-7), 505-524.

d'Ovidio, M. (2015). The field of fashion production in Milan: A theoretical discussion and an empirical investigation. City, Culture and Society, 6(2), 1-8.

Ponte, L. F., \& Campos, R. D. (2018). Taste Transformation in the Context of Social Mobility. BARBrazilian Administration Review, 15(2).

Quintao, R. T., Brito, E. P. Z., \& Belk, R. W. (2017). The taste transformation ritual in the specialty coffee market. Revista de Administração de Empresas, 57(5), 483-494.

Rafferty, K. (2011). Class-based emotions and the allure of fashion consumption. Journal of Consumer Culture, 11(2), 239-260.

Roling, L., \& Vieira, F. G. D. (2014). Interdisciplinaridade em Marketing: Perspectivas de aplicação dos conceitos teóricos de campo e habitus de Pierre Bourdieu às pesquisas em Marketing. Revista de Negócios, 19(3), 58-74. 
Saatcioglu, B., \& Ozanne, J. L. (2013). Moral habitus and status negotiation in a marginalized workingclass neighborhood. Journal of Consumer Research, 40(4), 692-710.

Shaw, E. H., \& Jones, D. B. (2005). A history of schools of marketing thought. Marketing Theory, 5(3), 239-281.

Sinclair, A. (2017). "It's a real negotiation within yourself": Women's stories of challenging heteronormativity within the habitus. Women's Studies International Forum, 64, 1-9.

Stamer, N. B. (2018). Moral conventions in food consumption and their relationship to consumers' social background. Journal of Consumer Culture, 18(1), 202-222.

Storey, M., Killian, S., \& O'Regan, P. (2017). Responsible management education: Mapping the field in the context of the SDGs. The International Journal of Management Education, 15(2), 93-103.

Strehlau, S. (2007). Alguns conceitos de Bourdieu e propostas de estudos em marketing. Anais XXXI Encontro da Associação Nacional dos Programas de Pós-Graduação em Administração Enanpad, Rio de janeiro.

Tapp, A., \& Warren, S. (2010). Field-capital theory and its implications for marketing. European Journal of Marketing, 44(1/2), 200-222.

Thiry-Cherques, H. R. (2006). Pierre Bourdieu: the theory in practice. Revista de Administração Pública, 40(1), 27-53.

Thompson, C. J., Arnould, E., \& Giesler, M. (2013). Discursivity, difference, and disruption: Genealogical reflections on the consumer culture theory heteroglossia. Marketing Theory, 13(2), 149-174.

Thompson, C. J., \& Üstüner, T. (2015). Women skating on the edge: Marketplace performances as ideological edgework. Journal of Consumer Research, 42(2), 235-265.

Tsaousi, C. (2016). 'What underwear do I like?'Taste and (embodied) cultural capital in the consumption of women's underwear. Journal of Consumer Culture, 16(2), 467-492.

Üstüner, T., \& Holt, D. B. (2009). Toward a theory of status consumption in less industrialized countries. Journal of Consumer Research, 37(1), 37-56.

Üstüner, T., \& Thompson, C. J. (2010). How marketplace performances produce interdependent status games and contested forms of symbolic capital. Journal of Consumer Research, 38(5), 796-814.

Vaidya, R. (2019). Corruption, re-corruption and what transpires in between: The case of a government officer in India. Journal of Business Ethics, 156(3), 605-620.

Yoganarasimhan, H. (2017). Identifying the presence and cause of fashion cycles in data. Journal of Marketing Research, 54(1), 5-26. 